\title{
Noise Fingerprints of Fiber Supercontinuum Sources
}

\author{
Dirk-Mathys Spangenberg ${ }^{1}$, Benoît Sierro ${ }^{1}$, Anupamaa Rampur ${ }^{1}$, Pascal Hänzi ${ }^{1}$, Alexander Hartung ${ }^{2}$, \\ Karol Tarnowski ${ }^{3}$, Pawel Mergo ${ }^{4}$, Tadeusz Martynkien ${ }^{3}$, Mariusz Klimczak ${ }^{5}$, and Alexander M. Heidt ${ }^{1}$ \\ ${ }^{1}$ Institute of Applied Physics, University of Bern, Sidlerstrasse 5, 3012 Bern, Switzerland \\ ${ }^{2}$ Leibniz-Institute of Photonic Technology, Albert-Einstein-Straße 9, D-07745 Jena, Germany \\ ${ }^{3}$ Dep. of Optics and Photonics, Wroclaw University of Science and Technology, Wybrzeze Wyspianskiego 27, 50-370 Wroclaw, Poland \\ ${ }^{4}$ Laboratory of Optical Fiber Technology, Maria Curie-Sklodowska University, pl. M. Curie-Sklodowskiej 3, 20-031 Lublin, Poland \\ ${ }^{5}$ Faculty of Physics, University of Warsaw, Pasteura 5, 02-093 Warsaw, Poland
}

Supercontinuum (SC) sources based on nonlinear spectral broadening of ultrashort pulses in specialty optical fibers have become an indispensable tool in a diverse range of application fields such as biomedical imaging, precision spectroscopy, and ultrafast photonics. After the initial race to maximize their spectral bandwidth and coverage, the noise properties of SC sources have recently shifted into focus. The large pulse-to-pulse fluctuations and correspondingly large relative intensity noise (RIN) present in commercial SC sources strongly limit the sensitivity, precision or resolution of many applications [1]. Recent work suggests that the dispersion engineering of the nonlinear fiber plays an important role for the resulting SC noise properties, as all-normal dispersion (ANDi) fibers suppress the noise-amplifying nonlinear processes that typically dominate the spectral broadening in their anomalously pumped counterparts $[2,3]$.

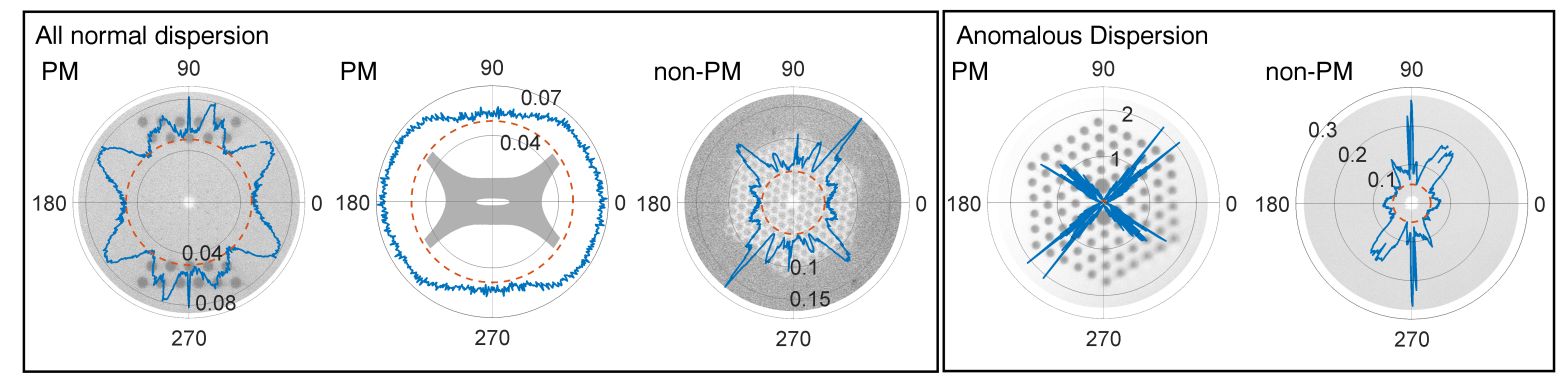

Fig. 1: Noise fingerprints of fiber SC sources covering the $1.2-2.2 \mu \mathrm{m}$ spectral region, implemented with several specialty nonlinear fibers exhibiting different cross-sectional geometries (shown in background) and dispersion profiles, both in polarizationmaintaing (PM) and non-PM versions. Polar plots show integrated RIN values (in \%) in dependence of input pulse polarization angle. The fiber's slow axis is aligned to zero degrees. The red-dotted line shows the RIN of the pump laser for comparison.

Here we present a novel technique measuring the noise properties of SC sources in unprecedented detail. For our experiments we inject pulses from an ultrafast Er:fiber laser ( $80 \mathrm{fs}, 40 \mathrm{MHz}, 0.05 \% \mathrm{RIN}$ ) into nonlinear fibers with various geometries and dispersion profiles, generating SC with comparable spectral bandwidths in the range $1.2-2.2 \mu \mathrm{m}$. A rotating half-wave plate in front of the fiber and a synchronized analyzer at the fiber exit control the plane of pump pulse and detection polarization with respect the fiber geometry. Polarization-dependent integrated RIN values from $0-18 \mathrm{MHz}$ are measured using a photodiode and electronic spectrum analyzer with an angular resolution of approximately $0.2^{\circ}$. The RIN values are visualized in polar plots that we refer to as "noise fingerprints" (Fig. 1), which are unique for a particular fiber SC source.

Since for each angular RIN measurement a full noise spectrum is available, we can identify the underlying noise amplification process for each feature of the fingerprint by analyzing its characteristic noise frequencies. Our analysis reveals that the geometry of the fiber strongly affects SC noise by inducing both unintentional and intentional residual stress in the manufacturing process. We identify several nonlinear polarization instability effects induced by unintentional stress in low-birefringence fibers, which lead to complex, highly polarization dependent noise amplification patterns. More regular and, therefore, predictable patterns emerge with highly birefringent fiber designs. While low-noise regions can be identified in all cases, highly birefringent ANDi fibers appear to be the most stable and environmentally robust sources of low-noise SC light, exhibiting RIN levels very similar to the pump laser even when the pump polarization does not align with a principal axis of the fiber. In contrast, the RIN of the anomalously pumped SC sources rises sharply for off-axis polarization reaching maximum RIN values of up to $2 \%$, which corresponds to a noise amplification factor of about 40 . Our results highlight the importance of birefringence and cross-sectional nonlinear fiber geometry, in addition to dispersion engineering, for the realization of high-quality, low-noise SC sources.

\section{References}

[1] S. Rao D.S., M. Jensen, L. Grüner-Nielsen, J. T. Olsen, P. Heiduschka, B. Kemper, J. Schnekenburger, M. Glud, M. Mogensen, N. Møller Israelsen, and O. Bang, "Shot-noise limited, supercontinuum based optical coherence tomography," arXiv:2010.05226 (2020).

[2] A. M. Heidt, J. S. Feehan, J. H. V. Price, and T. Feurer, "Limits of coherent supercontinuum generation in normal dispersion fibers," J. Opt. Soc. Am. B 34, 764-775 (2017).

[3] B. Sierro and A. M. Heidt, "Noise amplification in all-normal dispersion fiber supercontinuum generation and its impact in ultrafast photonics applications," OSA Continuum 3, 2347-2361 (2020). 